\title{
Efficient Gradient-Based Algorithm with Numerical Derivatives for Expedited Optimization of Multi-Parameter Miniaturized Impedance Matching Transformers
}

\author{
Slawomir KOZIEL ${ }^{1,2}$, Anna PIETRENKO-DABROWSKA ${ }^{2}$ \\ ${ }^{1}$ Engineering Optimization \& Modeling Center, Reykjavik University, Menntavegur 1, 101 Reykjavik, Iceland \\ ${ }^{2}$ Faculty of Electronics, Telecommunications and Informatics, Gdansk University of Technology, Narutowicza 11/12, \\ 80-233 Gdansk, Poland
}

koziel@ru.is, anna.dabrowska@pg.edu.pl

Submitted February 7, 2019 / Accepted June 13, 2019

\begin{abstract}
Full-wave electromagnetic (EM) simulation tools have become ubiquitous in the design of microwave components. In some cases, e.g., miniaturized microstrip components, EM analysis is mandatory due to considerable cross-coupling effects that cannot be accounted for otherwise (e.g., by means of equivalent circuits). These effects are particularly pronounced in the structures involving slow-wave compact cells and their numerical optimization is challenging due to expensive simulations and large number of parameters. In this paper, a novel gradient-based procedure with numerical derivatives is proposed for expedited optimization of compact microstrip impedance matching transformers. The method restricts the use of finite differentiation which is replaced for selected parameters by a rank-one Broyden updating formula. The usage of the formula is governed by an acceptance parameter which is made dependent on the parameter space dimensionality. This facilitates handling circuits of various complexities. The proposed approach is validated using three impedance matching transformer circuits with the number of parameters varying from ten to twenty. A significant speedup of up to 50 percent is demonstrated with respect to the reference algorithm.
\end{abstract}

\section{Keywords}

Microwave design closure, EM simulation, design optimization, trust-region framework, Broyden update, impedance transformers

\section{Introduction}

Small size has become a prerequisite in the design of many high-frequency structures, including RF, microwave and antenna components [1], [2]. The fundamental challenges come from physical limitations, e.g., some of the circuit dimensions being proportional to the guided wavelengths. These can be overcome to a certain extent by miniaturization methods such as transmission line folding [3], utilization of slow-wave compact cells [4], or various geometrical modifications (defected ground structures [5], stubs [6], radiator slots [7], etc.). Size reduction by topology alterations leads to practical problems related to parameter adjustment which have to be based on full-wave electromagnetic (EM) simulations. This is because neither analytical nor equivalent network models are able to account for cross-coupling effects in densely arranged layouts. Examples of relevant high-frequency structures include miniaturized microstrip couplers [8], impedance matching transformers [9], power dividers [10], filters [11], as well as antennas [12-14]. Another issue pertinent to compact components is an increased number of geometry variables. For example, a typical compact microstrip resonant cells (CMRC) unit is described by five or more parameters versus two for the conventional transmission line (TL) it is replacing [15]. Similarly, miniaturized antennas may require as many as twenty to thirty parameters to describe their geometry versus just a few for basic topologies. Finally, computational models of compact structures typically require longer simulation times (e.g., EM models of CMRC-based circuits normally feature higher-graded meshes than the TL-based ones). The abovementioned factors arising from device miniaturization i.e., cross-coupling effects, increased parameter number and lengthened simulation duration lead to excessive costs of the optimization process.

Below, the state-of the-art approaches for reducing the computational burden of EM-driven design closure are recited. One of the most straightforward methods is the use of adjoint sensitivities for expediting gradient-based routines [16]. Unfortunately, the availability of the adjoint technology is sparse in commercial simulation packages. A different approach is a reduction of the search space dimensionality, which can be achieved, among others, by 
means of reduced order models [17]. Yet another class of techniques are surrogate-based optimization (SBO) methods [18] which currently seem to be the most popular means of improving computational efficiency of simulation-driven design. One of the flagships of SBO in highfrequency engineering is space mapping (SM) [19]; other methods include manifold mapping [20], various response correction approaches (e.g., shape preserving response prediction [21], adaptive response scaling [22]), or featurebased optimization [23]). The most critical SBO component is the surrogate model, i.e., an auxiliary model which has to be significantly faster to evaluate than the original (high-fidelity) system representation. Practically utilized surrogates can be divided into two major groups: physicsbased, constructed by appropriate correction of the underlying low-fidelity models (e.g., equivalent networks [19]) and data-driven (also referred to as approximation ones, e.g., kriging [24], neural networks [25], polynomial chaos expansion [26]). Computational efficiency of SBO procedures is a combination of two factors: (i) shifting majority of operations into the surrogate model, and (ii) sparse evaluation of the high-fidelity model (executed for design verification and surrogate model enhancement). Unfortunately, the application of the aforementioned techniques for miniaturized microwave components faces serious difficulties. One of them is the lack of reliable low-fidelity models for compact structures. Yet another arises from the dimensionality issues: a typically large number of geometry parameters does not allow for constructing useable approximation surrogates. A possible workaround is a utilization of variable-fidelity EM models [27], however often coming at the price of significantly reduced computational efficiency of the optimization process.

The low-level optimization is typically performed using conventional methods. This is the case for direct design, where the subject of the optimization process is the high-fidelity EM model, but also for SBO procedures in which it is the surrogate model that is optimized in each iteration. In the context of local search, the methods of choice often include gradient-based routines [28] or derivative-free methods (e.g., pattern search [29]). The latter requires, however, many more objective function evaluations than gradient-based techniques [18]. In either case, reduction of the number of EM analyses required by the aforementioned low-level stage is fundamental for computational efficiency of the entire procedure.

In this paper, a trust-region gradient-based framework with numerical derivatives is proposed for reduced-cost optimization of compact microstrip impedance matching transformers in highly-dimensional parameter spaces. The technique described here allows for considerable reduction of the number of expensive EM simulations in the course of the optimization run by limiting the use of finite differentiation. This is achieved by a selective execution of the Broyden updating formula: it is only applied for the parameters for which the corresponding basis vectors are sufficiently well aligned with the direction of the recent design relocation ensuring by this this satisfactory accuracy of the resulting Jacobian estimate. In order to facilitate handling circuits of various complexities, the alignment acceptance threshold is made dependent on the search space dimensionality. The main scientific contributions of the proposed framework are: (i) a considerably reduced computational cost of the optimization process without significantly compromising the design quality by incorporation of the updating formulas into the Jacobian estimation process in a selective manner, (ii) delivering a way of automating the algorithm setup by making the alignment threshold dimensionality independent, (iii) suitability of the proposed algorithm to speed up both direct and surrogateassisted optimization procedures in the microwave area involving variable-fidelity models. For the sake of validation, several CMRC-based miniaturized impedance matching transformers are optimized, described by ten, fifteen, and twenty parameters, respectively. The results demonstrate considerable significant speedup (up to 50 percent, averaged over ten runs with random initial design) over the reference algorithm.

\section{Trust-Region Gradient-Search with Reduced Jacobian Updates}

This section provides the details on the design closure task formulation, the reference algorithm, as well as the proposed optimization algorithm with the emphasis put on the sensitivity update mechanisms.

\subsection{EM-Driven Design Closure}

Design closure is the last stage of the design process where the circuit topology is typically fixed and only the geometry (in some cases also material) parameter values are adjusted in order to boost the system performance as much as possible. If the task is dealt with using rigorous methods, e.g., numerical optimization, it is formulated as

$$
\boldsymbol{x}^{*}=\arg \min _{\boldsymbol{x}} U(\boldsymbol{R}(\boldsymbol{x}))
$$

in which $U$ is a scalar objective function, $\boldsymbol{R}(\boldsymbol{x})$ represents a response of the EM simulation model of the optimized structure, whereas $\boldsymbol{x}$ stands for a vector of adjustable parameters. Clearly, a definition of the function $U$ depends on the particular circuit, selected performance figures, and constraints imposed on the system. In this paper we focus on the impedance matching transformers, with the objective being reduction of the return loss within the frequency range of interest $F$. This can be formally written as

$$
U(\boldsymbol{R}(\boldsymbol{x}))=\max \left\{f \in F:\left|S_{11}(\boldsymbol{x}, f)\right|\right\} .
$$

In (2), the explicit dependence of the reflection coefficient $S_{11}$ on frequency $f$ is indicated for the sake of clarity. 


\subsection{Reference Algorithm}

As a reference algorithm a standard trust-region (TR) routine [30] is utilized and its concise recapitulation is given below. The TR framework is a convenient way of solving (1) if both the objective function and the constraints are evaluated through EM analysis. The routine delivers approximations to the optimum solution $\boldsymbol{x}^{*}$ as a series of vectors $\boldsymbol{x}^{(i)}, i=0,1, \ldots$

$$
\boldsymbol{x}^{(i+1)}=\arg \min _{\boldsymbol{x} ;-\boldsymbol{\delta}^{(i)} \leq \boldsymbol{x}-\boldsymbol{x}^{(i)} \leq \boldsymbol{\delta}^{(i)}} U\left(\boldsymbol{L}^{(i)}(\boldsymbol{x})\right)
$$

where $\boldsymbol{L}^{(i)}(\boldsymbol{x})=\boldsymbol{R}\left(\boldsymbol{x}^{(i)}\right)+\boldsymbol{J}_{\boldsymbol{R}}\left(\boldsymbol{x}^{(i)}\right) \cdot\left(\boldsymbol{x}-\boldsymbol{x}^{(i)}\right)$ represents a linear expansion of $\boldsymbol{R}$ at $\boldsymbol{x}^{(i)}$. In each iteration of the TR algorithm, $\boldsymbol{L}^{(i)}$ is determined by estimating the Jacobian $\boldsymbol{J}_{\boldsymbol{R}}$ through finite differentiation. For an $n$-element vector of the design variables of the structure at hand, it incurs a cost of additional $n$ EM simulations.

Here, unlike the conventional TR algorithm that adopts an Euclidean search region $\left\|\boldsymbol{x}-\boldsymbol{x}^{(i)}\right\| \leq \delta^{(i)}$, a hypercube-like search region is employed, and the inequalities $-\boldsymbol{\delta}^{(i)} \leq \boldsymbol{x}-\boldsymbol{x}^{(i)} \leq \boldsymbol{\delta}^{(i)}$ of (3) are interpreted as componentwise. This relates the initial TR region size, $\delta^{(0)}=\left\|\boldsymbol{\delta}^{(0)}\right\|$, with the design space sizes. This allows for accommodating the ranges of the geometry parameters (that typically vary from fractions of millimeters for gaps to tens of millimeters for longitudinal values).

\subsection{Trust-Region Optimization with Reduced Sensitivity Updates and Dimensionality- Dependent Alignment Threshold}

In the proposed algorithm, two mechanisms are simultaneously used to lessen the computational burden of sensitivity estimation. The first employs reduced sensitivity updates governed by the alignment of the recent design relocation with a given coordinate system axis. The second mechanism involves a flexible appointment of the alignment threshold value, which is both dimensionality- and convergence-dependent. Hence, the Jacobian $\boldsymbol{J}_{\boldsymbol{R}}$ estimation through FD, performed in each iteration of the conventional TR routine, is, for the chosen parameters, replaced with a rank-one Broyden formula (BF) [31]

$$
\boldsymbol{J}_{\boldsymbol{R}}^{(i+1)}=\boldsymbol{J}_{\boldsymbol{R}}^{(i)}+\frac{\left(\Delta \boldsymbol{R}^{(i+1)}-\boldsymbol{J}_{\boldsymbol{R}}^{(i)} \cdot \boldsymbol{h}^{(i+1)}\right) \cdot \boldsymbol{h}^{(i+1) \mathrm{T}}}{\boldsymbol{h}^{(i+1) \mathrm{T}} \boldsymbol{h}^{(i+1)}}
$$

where $\Delta \boldsymbol{R}^{(i+1)}=\boldsymbol{R}\left(\boldsymbol{x}^{(i+1)}\right)-\boldsymbol{R}\left(\boldsymbol{x}^{(i)}\right)=\left[\begin{array}{ll}\Delta R_{1}{ }^{(i)} & \ldots \Delta R_{n}{ }^{(i)}\end{array}\right]^{\mathrm{T}}$ and the recent design relocation vector is denoted as $\boldsymbol{h}^{(i+1)}=\boldsymbol{x}^{(i+1)}-\boldsymbol{x}^{(i)}=\left[h_{1}{ }^{(i)} \ldots h_{n}{ }^{(i)}\right]^{\mathrm{T}}$. The reason for applying the Broyden formula for the directions of search that are well aligned with the coordinate system axes is the following. The estimate $\boldsymbol{J}_{\boldsymbol{R}}{ }^{(i+1)}$ of the Jacobian is based on the design relocation between subsequent iterations $\boldsymbol{h}^{(i+1)}$ (cf. (4)). If $\boldsymbol{h}^{(i+1)}$ is well aligned with a particular coordinate system axis, the Broyden formula delivers a good approximation of the Jacobian in this direction, otherwise (4) does not bring any useful information into the Jacobian matrix and other means (here, FD) have to be used. It should be underlined, that in the proposed algorithm the entire Jacobian is estimated using FD merely in the first iteration, while in the subsequent iterations some (or all) of FD calculations may be replaced by the BF formula. The flow of the proposed algorithm with reduced sensitivity updates is as follows.

1. Set the iteration index $i=1$;

2. Calculate the entire Jacobian JR using FD;

3. Find the $\boldsymbol{x}^{(i+1)}$ by solving (3);

4. Update the TR region size;

5. Perform Jacobian update procedure (see Fig. 1);

6. If the termination condition is not satisfied go to 3 , else END.

The flow of the proposed Jacobian update procedure is shown graphically in Fig. 1. In its course, the Jacobian $\boldsymbol{J}_{\boldsymbol{R}}$ is merged from the columns $\boldsymbol{J}_{k}, k=1, \ldots, n$, either estimated with FD or computed with BF (cf. (4)). The variables, for which FD is to be skipped, are chosen based on the alignment between the recent design relocation direction and the coordinate system axes. For a given variable $k$ the decision factor $\varphi_{k}{ }^{(i)}$ is defined

$$
\varphi_{k}^{(i)}=\frac{\left|\boldsymbol{h}^{(i) \mathrm{T}} \boldsymbol{e}_{k}\right|}{\left\|\boldsymbol{h}^{(i)}\right\|} .
$$

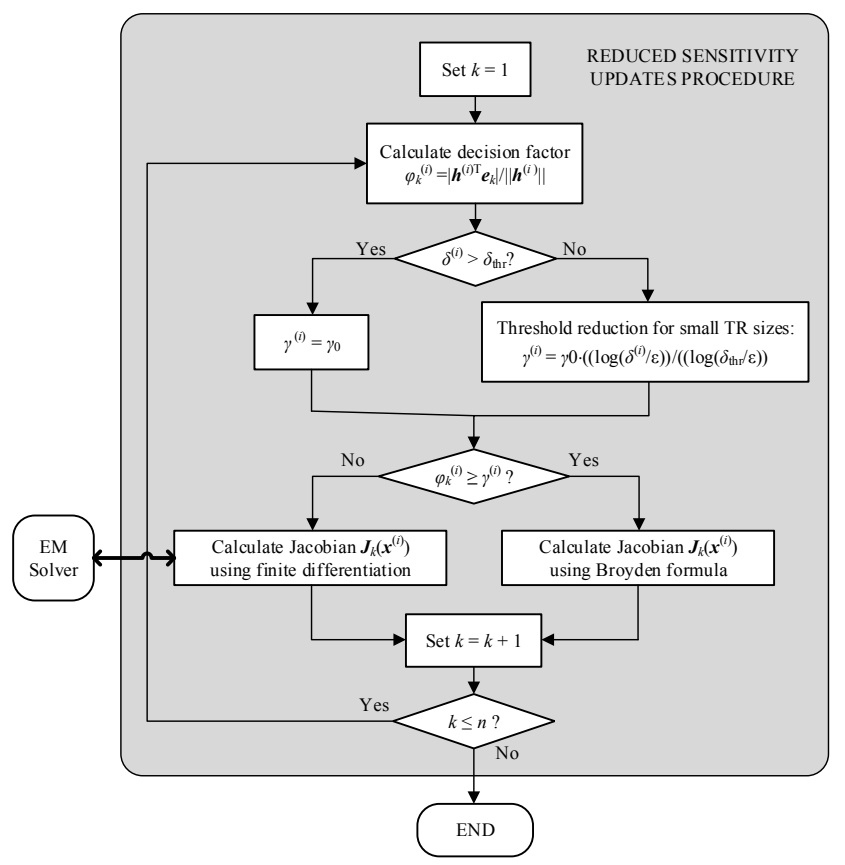

Fig. 1. Flow diagram of the reduced sensitivity updates procedure; the following notation is used: $\boldsymbol{J}_{k}=\partial R / \partial x_{k}-$ the $k$-th column of the Jacobian $\boldsymbol{J}_{\boldsymbol{R}} ; \varphi_{k}^{(i)}-$ a decision factor for the $i$-th iteration and for the $k$ th parameter, $k=1$, $\ldots, n ; \boldsymbol{e}_{k}=\left[e_{k, 1} \ldots e_{k, n}\right]^{\mathrm{T}}-$ standard basis vectors $\left(e_{k, j}=0\right.$ for $k \neq j$, with 1 on the $k$-th position); $\delta^{(i)}=\left\|\delta^{(i)}\right\|-$ a TR region size in the $i$-th iteration, $\delta_{\text {thr }}-$ a user-defined TR region size threshold value; $\varepsilon-$ a termination condition (for convergence in argument); $\gamma_{0}-$ an initial alignment acceptance threshold value; $\gamma_{k}^{(i)}-$ an alignment threshold value in the $i$-th iteration. 


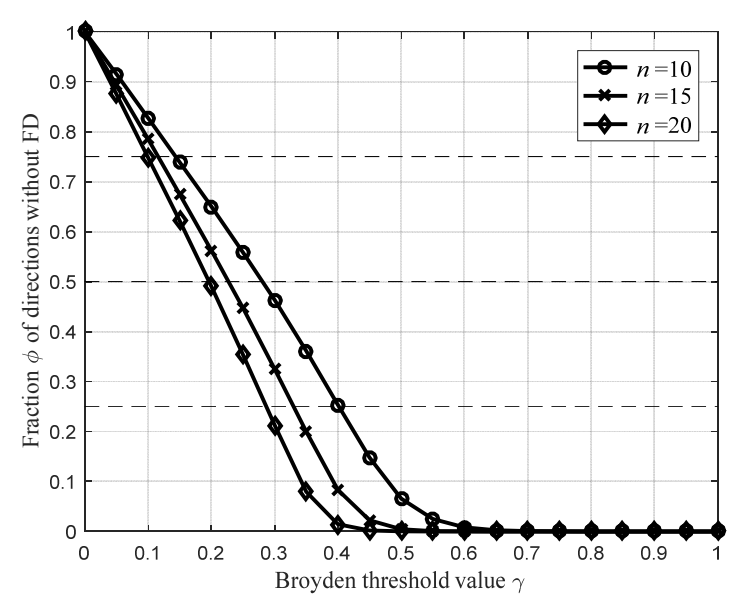

Fig. 2. The assumed fraction $\phi$ of directions without FD versus the threshold value $\gamma$ for $n=10,15$ and 20 equal to the number of the design variables for two-, three- and four-section transformer, respectively, as described in Sec. 3 .

If the alignment exceeds the alignment acceptance threshold value $\gamma_{k}^{(i)}$, the respective Jacobian column $\boldsymbol{J}_{k}$ is calculated using BF; otherwise FD is executed. The above definition of the decision factor implies that $\varphi_{k}^{(i)}=1$ if the design relocation vector $\boldsymbol{h}^{(i)}$ and the $k$-th basis vector $\boldsymbol{e}_{k}$ are collinear and $\varphi_{k}^{(i)}=0$ if the vectors are orthogonal. Here, the current threshold value $\gamma^{(i)}$ for the $i$-th iteration is contingent on the current TR region size $\delta^{(i)}$ being the algorithm convergence status. In the $i$-th iteration, $\gamma^{(i)}$ is calculated as

$$
\gamma^{(i)}=\gamma_{0}\left(\left(\log \left(\delta^{(i)} / \varepsilon\right)\right) /\left(\log \left(\delta_{\mathrm{thr}} / \varepsilon\right)\right)\right)
$$

where $0 \leq \gamma_{0} \leq 1$ is a user defined initial threshold value (algorithm control parameter), $\delta_{\text {thr }}$ is a boundary value of the TR region size, and $\varepsilon$ refers to a termination condition (for convergence in argument). The choice of $\gamma_{\text {init }}$ is a crucial one, as it strongly influences the optimization process. The higher $\gamma_{\text {init }}$, the higher the overall number of FD estimations associated with a lengthened optimization process duration and, at the same time, potentially better design quality. If the low computational cost is of primary importance, then lower $\gamma_{\text {init }}$ values are preferred. In order to secure higher computational savings, the progressive reduction of the acceptance threshold value is carried out that leads to an even less frequent utilization of FD. As the algorithm converges, the TR region size $\delta^{(i)}$ is reduced according to the standard rules [31]. In the case of the small TR region size, below user-defined boundary value $\delta_{\text {thr }}$, the alignment threshold value $\gamma^{(i)}$ is gradually reduced, tending to 0 as $\delta^{(i)}$ approaches $\varepsilon$.

The idea of making dimension-dependent value of the acceptance threshold $\gamma_{\text {init }}$ makes the algorithm setup dimensionality independent (see Sec. 3). In the paper, $\gamma_{\text {init }}$ is calculated based on the number of the design variables of the structure at hand. First, the requested fraction $\phi$ of the Jacobian columns without FD is selected. Then, the actual acceptance threshold value $\gamma_{\phi}$, that permits attaining $\phi$, is obtained using statistical optimization. The following problem needs to be solved

$$
\gamma_{\phi}=\arg \min _{\gamma}\left|\phi-N_{\phi}^{-1} \sum_{j=1}^{N_{\phi}} \sum_{k=1}^{n}\left[\beta_{j . k}-\gamma\right]\right| .
$$

Here, for a given problem dimensionality $n$, let us denote the statistical factors $\beta_{j . k}=\left|\boldsymbol{y}^{(k) \mathrm{T}} \boldsymbol{e}^{(k)}\right| / \mid \boldsymbol{y}^{(k)} \|$, for $j=1, \ldots$, $N_{\phi}$ and $k=1, \ldots, n$, where $\boldsymbol{y}^{(j)}, j=1, \ldots, N_{\phi}$, refer to a series of random observations. The problem of optimizing the fraction of directions, for which the coefficient is over $\gamma$, is solved using a pattern search algorithm [29].

Figure 2 shows the relation between the desired fraction $\phi$ of directions without FD and the threshold value $\gamma$ depicted for the parameter space dimensionalities corresponding to those of the transformers from Sec. 3. Different values of $n$ are associated with various threshold values securing the same fraction of savings. The dimension-dependent threshold allows us for achieving a consistent algorithm performance for the structures described by different number of parameters. In particular, the optimum value of the fraction $\phi$ appears to be dimensionality independent as validated by the results from Tab. 2 .

\section{Numerical Validation}

In this section, a comprehensive validation of the framework introduced in Sec. 2 is provided. The primary purpose is the evaluation of potential computational savings that can be achieved over the reference method (Sec. 2.2). Other goals include investigation of the algorithm robustness, which is realized by means of multiple optimization runs involving random initial designs. Finally, the method scalability is verified by considering benchmark cases of various dimensionalities.

\subsection{Benchmark Structures}

The verification structures are three miniaturized impedance matching transformers shown in Fig. 3. The circuits

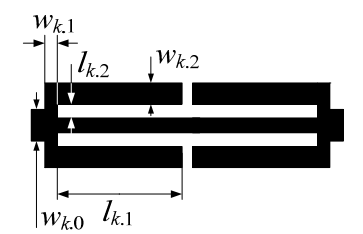

(a)

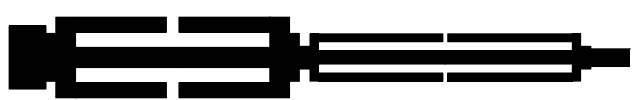

(b)

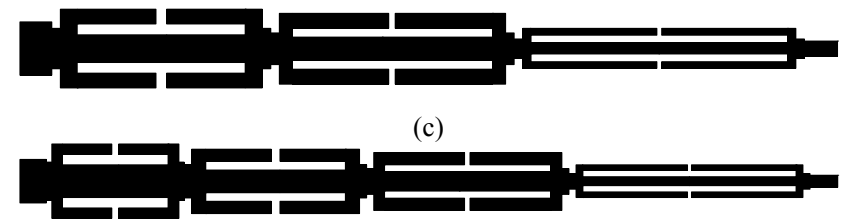

(d)

Fig. 3. Verification test cases: (a) CMRC cell, (b)-(d) CMRCbased miniaturized two-, three- and four-section impedance matching transformers. 


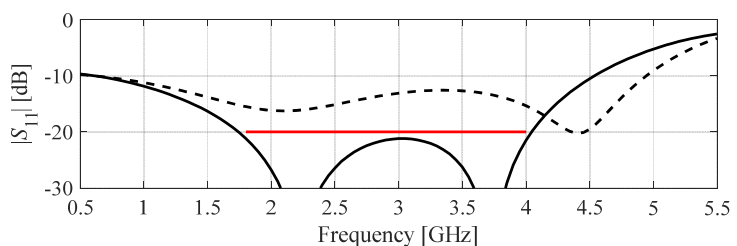

(a)

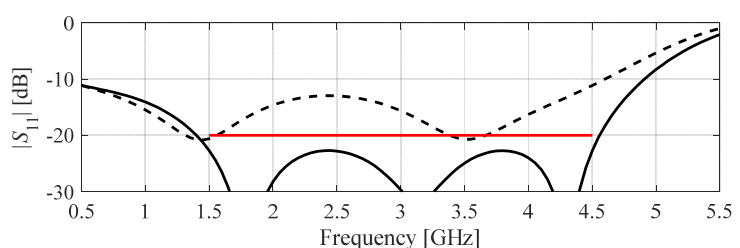

(b)

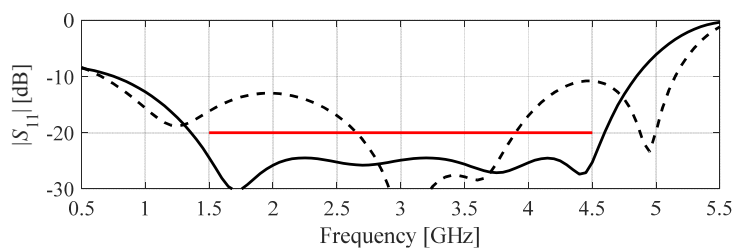

(c)

Fig. 4. Initial (- - -) and optimized (-) responses of the impedance matching transformers at the selected designs obtained using the proposed methodology: (a) two-section transformer, (b) three-section transformer, (c) four-section transformer. Intended operational bandwidth marked using horizontal lines.

\begin{tabular}{|c|c|c|c|c|}
\hline \multirow{2}{*}{ Circuit } & \multicolumn{3}{|c|}{ Specifications $^{*}$} & \multirow{2}{*}{ Geometry Parameters } \\
\cline { 2 - 4 } & $Z_{1}[\Omega]$ & $Z_{2}[\Omega]$ & $B[\mathrm{GHz}]$ & \\
\hline 2-section & 50 & 100 & $1.5-4.5$ & $\begin{array}{l}{\left[l_{1.1} l_{1.2} w_{1.1} w_{1.2} w_{1.0}\right.} \\
\left.l_{2.1} l_{2.2} w_{2.1} w_{2.2} w_{2.0}\right]^{\mathrm{T}}\end{array}$ \\
\hline 3-section & 50 & 100 & $1.5-4.5$ & $\begin{array}{l}{\left[l_{1.1} l_{1.2} w_{1.1} w_{1.2} w_{1.0}\right.} \\
l_{2.1} l_{2.2} w_{2.1} w_{2.2} w_{2.0} \\
\left.l_{3.1} l_{3.2} w_{3.1} w_{3.2} w_{3.0}\right]^{\mathrm{T}}\end{array}$ \\
\hline 4-section & 50 & 130 & $2.0-4.0$ & $\begin{array}{l}{\left[l_{1.1} l_{1.2} w_{1.1} w_{1.2} w_{1.0}\right.} \\
l_{2.1} l_{2.2} w_{2.1} w_{2.2} w_{2.0} \\
l_{3.1} l_{3.2} w_{3.1} w_{3.2} w_{3.0} \\
\left.l_{4.1} l_{4.2} w_{4.1} w_{4.2} w_{4.0}\right]^{\mathrm{T}}\end{array}$ \\
\hline
\end{tabular}

Tab. 1. Impedance transformers: parameters and specs. * $Z_{1}$ and $Z_{2}$ stand for the input and load impedance, respectively; $B$ is the operating band.

are implemented on a 0.762-mm-thick Taconic RF35 substrate $\left(\varepsilon_{\mathrm{r}}=3.5\right)$. All structures are based on compact microstrip resonant cells (CMRCs) shown in Fig. 3(a). Table 1 provides information about the geometry parameters as well as the design specifications. Our objective is to minimize the reflection coefficient in the frequency band $B$ as described in the table.

\subsection{Numerical Results}

The transformers of Fig. 3 have been optimized according to the specifications of Tab. 1 using the proposed algorithm as well as the reference TR procedure. In order to obtain more comprehensive data, especially in terms of the robustness, the algorithms were executed ten times starting from random initial designs. Tables 2 and 3 gather the relevant numerical data. For illustration purposes, Figure 4 shows the initial and optimized transformer re- sponses at the selected designs. The lower bounds for design variables of the four-section transformer are $\boldsymbol{l}_{4}=[2$

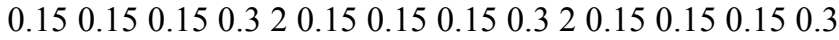

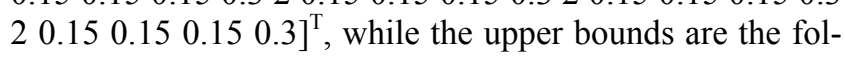

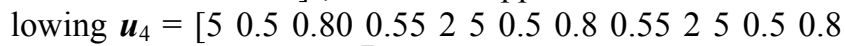

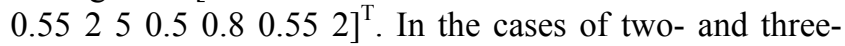
section transformer, the lower and upper bounds are $\boldsymbol{l}_{2}=\boldsymbol{l}_{4}$ $(1: 10), \boldsymbol{u}_{2}=\boldsymbol{u}_{4}(1: 10)$ and $\boldsymbol{l}_{3}=\boldsymbol{l}_{4}(1: 15), \boldsymbol{u}_{3}=\boldsymbol{u}_{4}(1: 15)$, respectively.

\subsection{Discussion}

The results presented in Tab. 2 and 3 confirm that the algorithm of Sec. 2 permits achieving substantial computational speedup of the optimization process without a significant loss of the design quality. The following values of the fraction of the directions without FD were considered $\phi=0.6,0.8,0.9,0.95,0.98$ and 1.0. Clearly, increasing $\phi$

\begin{tabular}{|c|c|c|c|c|c|c|c|}
\hline \multirow{3}{*}{\multicolumn{2}{|c|}{ Algorithm }} & \multicolumn{6}{|c|}{ Transformer } \\
\hline & & \multicolumn{2}{|c|}{ Two-section } & \multicolumn{2}{|c|}{ Three-section } & \multicolumn{2}{|c|}{ Four-section } \\
\hline & & $\operatorname{Cost}^{*}$ & $\begin{array}{l}\max \\
\left|S_{11}\right|^{\#} \\
{[\mathrm{~dB}]}\end{array}$ & $\operatorname{Cost}^{*}$ & $\begin{array}{l}\max \\
\left|S_{11}\right|^{\#} \\
{[\mathrm{~dB}]}\end{array}$ & $\operatorname{Cost}^{*}$ & $\begin{array}{l}\max \\
\left|S_{11}\right|^{\#} \\
{[\mathrm{~dB}]}\end{array}$ \\
\hline \multicolumn{2}{|c|}{$\begin{array}{l}\text { Reference } \\
\text { algorithm }\end{array}$} & 95.0 & -21.4 & 160.0 & -21.9 & 176.8 & -25.3 \\
\hline \multirow{6}{*}{ 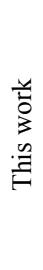 } & $\phi=0.6$ & 77.5 & -21.4 & 115.6 & -21.6 & 143.9 & -25.0 \\
\hline & $\phi=0.8$ & 74.6 & -21.3 & 100.5 & -21.7 & 105.8 & -24.1 \\
\hline & $\phi=0.9$ & 47.0 & -21.0 & 113.6 & -21.8 & 89.6 & -24.9 \\
\hline & $\phi=0.95$ & 52.7 & -21.0 & 95.9 & -21.7 & 87.4 & -23.7 \\
\hline & $\phi=0.98$ & 49.9 & -20.9 & 76.6 & -21.5 & 69.0 & -23.5 \\
\hline & $\phi=1.0$ & 34.0 & -20.7 & 76.6 & -21.4 & 62.7 & -23.3 \\
\hline
\end{tabular}

Tab. 2. Optimization results and benchmarking: * Number of EM simulations averaged over 10 algorithm runs (random initial points). \# Maximum $\left|S_{11}\right|$ within the intended operating bandwidth (averaged over 10 algorithm runs).

\begin{tabular}{|c|c|c|c|c|c|c|c|c|c|c|}
\hline \multirow{3}{*}{\multicolumn{2}{|c|}{ Algorithm }} & \multicolumn{9}{|c|}{ Transformer } \\
\hline & & \multicolumn{3}{|c|}{ Two-section } & \multicolumn{3}{|c|}{ Three-section } & \multicolumn{3}{|c|}{ Four-section } \\
\hline & & 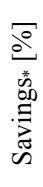 & 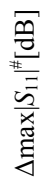 & $\begin{array}{l}\text { 吾 } \\
\text { 是 }\end{array}$ & 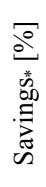 & 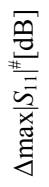 & 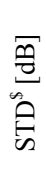 & 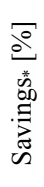 & 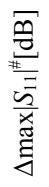 & $\begin{array}{l}\bar{q} \\
\text { 吾 } \\
\text { 是 }\end{array}$ \\
\hline \multicolumn{2}{|c|}{$\begin{array}{l}\text { Reference } \\
\text { algorithm }\end{array}$} & - & - & 0.2 & - & - & 0.6 & - & - & 1.1 \\
\hline \multirow{6}{*}{ 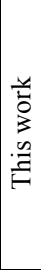 } & $\phi=0.6$ & 18 & 0.0 & 0.2 & 28 & 0.3 & 0.8 & 19 & 0.3 & 1.2 \\
\hline & $\phi=0.8$ & 21 & 0.1 & 0.2 & 37 & 0.2 & 0.8 & 40 & 1.2 & 1.2 \\
\hline & $\phi=0.9$ & 50 & 0.3 & 0.3 & 29 & 0.1 & 0.5 & 49 & 0.4 & 1.2 \\
\hline & $\phi=0.95$ & 45 & 0.3 & 0.3 & 40 & 0.2 & 0.6 & 50 & 1.6 & 1.5 \\
\hline & $\phi=0.98$ & 47 & 0.5 & 0.5 & 52 & 0.4 & 0.9 & 61 & 1.8 & 1.4 \\
\hline & $\phi=1.0$ & 64 & 0.7 & 0.6 & 52 & 0.5 & 1.1 & 65 & 2.0 & 1.8 \\
\hline
\end{tabular}

Tab. 3. Computational savings and design quality: * Percentage-wise cost savings w.r.t. the reference algorithm. \# Degradation of objective function value w.r.t. the reference algorithm. ${ }^{\$}$ Standard deviation of the objective function over all algorithm runs. 
leads to higher computational savings and, at the same time, certain deterioration of the design quality.

The quality degradation is minor (as compared to the reference algorithm) for all three transformers as long as $\phi$ does not exceed 0.95 . It should be noted that a proper comparison between the reference and the proposed algorithm should take into consideration non-zero standard deviation for the reference procedure, which is a result of using random initial designs leading to various local optima. Consequently, it is the difference between the standard deviations of the proposed algorithm (for a given fraction $\phi$ ) and the reference one that indicates the results repeatability degradation. It increases considerably for $\phi$ beyond 0.95 . The fraction of $\phi=0.9$ appears to be the best from the point of view of the cost-accuracy trade-off. This value ensures, across the benchmark set, the savings as high as $50 \%$ (for two-section transformer) accompanied with an insignificant quality decline (below $0.5 \mathrm{~dB}$ ). It should be noticed, that the declared fraction value is dimensionality independent which is an advantageous feature of the proposed technique in the context of the algorithm setup.

\section{Conclusion}

The purpose of this paper was to introduce a reducedcost trust-region gradient-search framework with numerical derivatives. The intended use of the procedure is design closure of CMRC-based impedance matching transformers. These circuits are typically characterized by a relatively large number of geometry parameters, which incurs considerable optimization cost. The proposed methodology has been comprehensively validated and demonstrated to yield a considerable reduction of the computational cost of the optimization process. The savings are as high as 50 percent as compared to the reference (finite-differentiation-based) algorithm. Furthermore, the acceptance threshold for applying the sensitivity updating formula is made dimensionality dependent, which results in the trade-offs between the cost reduction and quality degradation being consistent for various parameter space dimensionalities (here, from ten to twenty). Consequently, setting up the algorithm control parameters is straightforward. Although demonstrated for impedance matching transformers, other foreseeable applications of the framework are possible and include direct EM-driven design closure of other compact microwave components but also optimization of corrected low-fidelity EM models within surrogate-assisted procedures.

\section{Acknowledgments}

The authors would like to thank Dassault Systemes, France, for making CST Microwave Studio available. This work is partially supported by the Icelandic Centre for Research (RANNIS) Grant 174114051 and by National Science Centre of Poland Grant 2015/17/B/ST6/01857.

\section{References}

[1] CARiou, M., POTElon, B., QUENDO, C., et al. Compact Xband filter based on substrate integrated coaxial line stubs using advanced multilayer PCB technology. IEEE Transactions on Microwave Theory and Techniques, 2017. vol. 65, no. 2, p. 496-503. DOI: 10.1109/TMTT.2016.2632114

[2] FUJiMOTO, K., MORISHITA, H. Modern Small Antennas. Cambridge (UK): Cambridge University Press, Cambridge, 2014. ISBN: 978-0-521-87786-2, DOI: 10.1017/CBO9780511977602

[3] TSENG, C.-H., CHEN, H.-J. Compact rat-race coupler using shunt-stub-based artificial transmission lines. IEEE Microwave and Wireless Components Letters, 2008, vol. 18, no. 11, p. 734-736. DOI: 10.1109/LMWC.2008.2005225

[4] KOZIEL, S., KURGAN P. Inverse modeling for fast design optimization of small-size rat-race couplers incorporating compact cells. International Journal of RF \& Microwave Computer Aided Engineering, 2018, vol. 28, no. 5. DOI: 10.1002/mmce.21240

[5] MAO, Y., GUO, S., CHEN, M. Compact dual-band monopole antenna with defected ground plane for Internet of Things. IET Microwaves, Antennas and Propagation, 2018, vol. 12, no. 8, p. 1332-1338. DOI: 10.1049/iet-map.2017.0860

[6] LI, W., HEI, Y., GRUBB, P. M., et al. Compact inkjet-printed flexible MIMO antenna for UWB applications. IEEE Access, 2018, vol. 6, p. 50290-50298. DOI: 10.1109/ACCESS.2018.2868707

[7] TANG, H., WANG, K. WU, R., et al. A novel broadband circularly polarized monopole antenna based on $\mathrm{C}$-shaped radiator. IEEE Antennas and Wireless Propagation Letters, 2017, vol. 16, p. 964-967. DOI: 10.1109/LAWP.2016.2615159

[8] TING, H. L., HSU, S. K., WU, T. L. A novel and compact eightport forward-wave directional coupler with arbitrary coupling level design using four-model control theory. IEEE Transactions on Microwave Theory and Techniques, 2017, vol. 65, no. 2, p. 467-475. DOI: 10.1109/TMTT.2016.2623709

[9] KOZIEL, S., BEKASIEWICZ, A. Rapid simulation-driven multiobjective design optimization of decomposable compact microwave passives. IEEE Transactions on Microwave Theory and Techniques, 2016, vol. 64, no. 8, p. 2454-2461. DOI: 10.1109/TMTT.2016.2583427

[10] KHAN, A. A., MANDAL, M. K. Miniaturized substrate integrated waveguide (SIW) power dividers. IEEE Microwave and Wireless Components Letters, 2016, vol. 26, no. 11, p. 888-890. DOI: 10.1109/LMWC.2016.2615005

[11] SHEIKHI, A., ALIPOUR, A., ABDIPOUR, A. Design of compact wide stopband microstrip low-pass filter using T-shaped resonator. IEEE Microwave and Wireless Components Letters, 2017, vol. 27, no. 2, p. 111-113. DOI: 10.1109/LMWC.2017.2652862

[12] LI, W., TU, Z., CHU, Q., et al. Differential stepped-slot UWB antenna with common-mode suppression and dual sharp-selectivity notched bands. IEEE Antennas and Wireless Propagation Letters, 2016, vol. 11, p. 1120-1123. DOI: 10.1109/LAWP.2015.2496159

[13] PANDEY, G. K., VERMA, H., MESHRAM, M. K. Compact antipodal Vivaldi antenna for UWB applications. Electronics Letters, 2015, vol. 51, no. 4, p. 308-310. DOI: $10.1049 / \mathrm{el} .2014 .3540$

[14] SRIVAStaVA, G., MOHAN, A., CHAKRABARTY, A. Compact reconfigurable UWB slot antenna for cognitive radio applications. IEEE Antennas and Wireless Propagation Letters, 2016, vol. 16, p. 1139-1142. DOI: 10.1109/lawp.2016.2624736

[15] KOZIEL, S., KURGAN, P. Compact cell topology selection for size-reduction-oriented design of microstrip rat-race couplers. International Journal of $R F \&$ Microwave Computer Aided Engineering, 2018, vol. 28, no. 5. DOI: 10.1002/mmce.21261 
[16] ZHANG, Y., NIKOLOVA, N. K., MESHRAM, M. K. Design optimization of planar structures using self-adjoint sensitivity analysis. IEEE Transactions on Antennas and Propagation, 2012, vol. 60, no. 6, p. 3060-3066. DOI: 10.1109/TAP.2012.2194684

[17] BURGARD, S., FARLE, O., LOEW, P. Fast shape optimization of microwave devices based on parametric reduced-order models. IEEE Transactions on Magnetics, 2014, vol. 50, no. 2, p. 629-632. DOI: 10.1109/TMAG.2013.2282420

[18] KOZIEL, S., YANG, X. S., ZHANG, Q. J. (Eds.) SimulationDriven Design Optimization and Modeling for Microwave Engineering. London (UK): Imperial College Press, 2013. ISBN: 978-1848169166 DOI: 10.1142/p860

[19] BANDLER, J. W., CHENG, Q. S., DAKROURY, S. A., et al. Space mapping: the state of the art. IEEE Transactions on Microwave Theory and Techniques, 2004, vol. 52, no. 1, p. 337-361. DOI: 10.1109/TMTT.2003.820904

[20] SU, Y., LI, J., FAN, Z., et al. Shaping optimization of double reflector antenna based on manifold mapping. In International Applied Computational Electromagnetics Society Symposium (ACES). Shuzou (China), 2017, p. 1-2. ISBN: 978-0-9960-0785-6

[21] LEIFSSON, L., KOZIEL, S. Surrogate modeling and optimization using shape-preserving response prediction: a review. Engineering Optimization, 2014, vol. 48, no. 3, p. 476-496. DOI: 10.1080/0305215X.2015.1016509

[22] KOZIEL, S., BEKASIEWICZ, A. Rapid microwave design optimization in frequency domain using adaptive response scaling. IEEE Transactions on Microwave Theory and Techniques, 2016 , vol. 64, no. 9, p. 2749-2757. DOI: 10.1109/TMTT.2016.2590551

[23] KOZIEL, S. Fast simulation-driven antenna design using responsefeature surrogates. International Journal of $R F \&$ Microwave Computer Aided Engineering, 2015, vol. 25, no. 5, p. 394-402. DOI: $10.1002 /$ mmce. 20873

[24] DE VILliERS, D. I. L., COUCKUYT, I., DHAENE, T. Multiobjective optimization of reflector antennas using kriging and probability of improvement. In IEEE AP-S International Symposium on Antennas and Propagation. San Diego (USA), 2017, p. 985-986, DOI: 10.1109/APUSNCURSINRSM.2017.8072535

[25] ZHANG, C., JIN, J., NA, W., et al. Multivalued neural network inverse modeling and applications to microwave filters. IEEE Transactions on Microwave Theory and Techniques, 2018, vol. 66, no. 8, p. 3781-3797. DOI: 10.1109/TMTT.2018.2841889

[26] ZHANG, J., ZHANG, C., FENG, F., et al. Polynomial chaos-based approach to yield-driven EM optimization. IEEE Transactions on Microwave Theory and Techniques, 2018, vol. 66, no. 7, p. 3186-3199. DOI: 10.1109/TMTT.2018.2834526

[27] KOZIEL, S., KURGAN, P. Rapid design of miniaturized branchline couplers through concurrent cell optimization and surrogate- assisted fine-tuning. IET Microwaves, Antennas and Propagation, 2015, vol. 9, no. 9, p. 957-963. DOI: 10.1049/iet-map.2014.0600

[28] NOCEDAL, J., WRIGHT, S. J. Numerical Optimization. 2nd ed. New York (USA): Springer, 2006. ISBN: 978-0-387-40065-5 DOI: 10.1007/978-0-387-40065-5

[29] KOZIEL, S. Computationally efficient multi-fidelity multi-grid design optimization of microwave structures. Applied Computational Electromagnetics Society Journal, 2010, vol. 25, no. 7 , p. $578-586$.

[30] CONN, A. R., GOULD, N. I. M., TOINT, P. L. Trust Region Methods. Philadelphia (USA): MPS-SIAM Series on Optimization, 2000. ISBN: 0-89871-460-5 DOI: 10.1137/1.9780898719857

[31] BROYDEN, C. G. A class of methods for solving nonlinear simultaneous equations. Mathematics of Computation, 1965, vol. 19, no. 92, p. 577-593. DOI: 10.1090/S0025-5718-19650198670-6

\section{About the Authors ...}

Slawomir KOZIEL received the M.Sc. and Ph.D. degrees in Electronic Engineering from Gdansk University of Technology, Poland, in 1995 and 2000, respectively. He also received the M.Sc. degrees in Theoretical Physics and in Mathematics, in 2000 and 2002, respectively, as well as the Ph.D. in Mathematics in 2003, from the University of Gdansk, Poland. He is currently a Professor with the School of Science and Engineering, Reykjavik University, Iceland. His research interests include CAD and modeling of microwave and antenna structures, simulation-driven design, surrogate-based optimization, space mapping, circuit theory, analog signal processing, evolutionary computation and numerical analysis.

Anna PIETRENKO-DABROWSKA (corresponding author) received the M.Sc. and Ph.D. degrees in Electronic Engineering from Gdansk University of Technology, Poland, in 1998 and 2007, respectively. Currently, she is an Associate Professor with Gdansk University of Technology, Poland. Her research interests include simulationdriven design, design optimization, control theory, modeling of microwave and antenna structures, numerical analysis. 